\title{
Accurate classification of membrane protein types based on sequence and evolutionary information using deep learning
}

\author{
Lei Guo ${ }^{1 \dagger}$, Shunfang Wang ${ }^{1 *}{ }^{*}$, Mingyuan $\mathrm{Li}^{1}$ and Zicheng Cao ${ }^{2}$
}

From 2018 International Conference on Intelligent Computing (ICIC 2018) and Intelligent Computing and Biomedical Informatics (ICBI) 2018 conference

Wuhan and Shanghai, China. 15-18 August 2018, 3-4 November 2018

\begin{abstract}
Background: Membrane proteins play an important role in the life activities of organisms. Knowing membrane protein types provides clues for understanding the structure and function of proteins. Though various computational methods for predicting membrane protein types have been developed, the results still do not meet the expectations of researchers.

Results: We propose two deep learning models to process sequence information and evolutionary information, respectively. Both models obtained better results than traditional machine learning models. Furthermore, to improve the performance of the sequence information model, we also provide a new vector representation method to replace the one-hot encoding, whose overall success rate improved by $3.81 \%$ and $6.55 \%$ on two datasets. Finally, a more effective model is obtained by fusing the above two models, whose overall success rate reached $95.68 \%$ and $92.98 \%$ on two datasets.

Conclusion: The final experimental results show that our method is more effective than existing methods for predicting membrane protein types, which can help laboratory researchers to identify the type of novel membrane proteins.
\end{abstract}

Keywords: Deep learning, Membrane protein type prediction, Vector representation

\section{Background}

Protein is an important component of all cells and tissues in the human body and is the material basis of life [1-5]. Membrane proteins represent one important protein type that is rich in function, they participate in many important reactions of the cell, including transporting substances into and out of cells as a carrier, acting as a

\footnotetext{
*Correspondence: sfwang_66@ynu.edu.cn

†Lei Guo and Shunfang Wang contributed equally to this work. ${ }^{1}$ Department of Computer Science and Engineering, School of Information Science and Engineering, Yunnan University, Kunming 650504, People's Republic of China

Full list of author information is available at the end of the article
}

specific receptor for hromones, and carrying out cellular recognition functions, as well as being responsible for signal transduction and cell-cell interactions [6]. Among the genomes that have been completely sequenced, the number of membrane proteins accounts for 30\% [7]. Moreover, membrane proteins are of particular importance in drug therapy, as they act as the targets for many drugs. Currently, the effects more than $50 \%$ of drugs on the market are exerted by the actions of membrane proteins [8]. Since the function of membrane protein is closely related to its type, the prediction of membrane protein types can contribute to research in the field of bioinformatics.

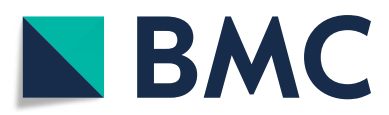

(c) The Author(s). 2019 Open Access This article is distributed under the terms of the Creative Commons Attribution 4.0 International License (http://creativecommons.org/licenses/by/4.0/), which permits unrestricted use, distribution, and reproduction in any medium, provided you give appropriate credit to the original author(s) and the source, provide a link to the Creative Commons license, and indicate if changes were made. The Creative Commons Public Domain Dedication waiver (http://creativecommons.org/publicdomain/zero/1.0/) applies to the data made available in this article, unless otherwise stated. 
According to their functions, membrane proteins can be classified into three classes: integral, peripheral and lipidanchored [9]. Based on the direct interaction relationship between membrane proteins and lipid bilayers, the three classes can be further extended into eight basic types: (1) type I membrane proteins, (2) type II membrane proteins, (3) type III membrane proteins (4) type IV membrane proteins, (5) multipass transmembrane proteins, (6) lipid chain-anchored membrane proteins, (7) GPI-anchored membrane proteins, and (8) peripheral membrane proteins. Among them, Types I, II, III and IV are singlepass transmembrane proteins, and detailed descriptions of their differences are given in [9].

To help laboratory researchers discover the type of novel membrane protein, various computation methods are proposed for membrane protein type recognition. Many of these approaches incorporate machine learning algorithms and statistical analysis techniques, such as knearest neighbor (KNN) [10], the naive Bayesian model (NBM) [11], support vector machines (SVM) [12-14], random forests (RF) [15], probabilistic neural network (PNN) [16] and hidden Markov models [7].

The most popular feature extraction methods for predicting membrane protein types are based on sequence information. The pseudo-amino acid composition (PseAAC) [17] method incorporates information about sequence order. Local amino acid composition (LAAC), local dipeptide composition (LDC), global descriptor (GD), Lempel-Ziv complexity (LZC), autocorrelation descriptor (AD), sequence-order descriptor (SD) and Hilbert-Huang transform (HHT) are proposed based on amino acid classification and physicochemical properties [18], and these methods are partially applied in our previous research [19]. The peptide composition method, such as dipeptide composition (DipC) [20] and tripeptide composition (TipC) [21] are also powerful sequence information-based feature extraction methods.

The evolutionary information, which mainly refers to the position-specific scoring matrix (PSSM), is also widely used to predict membrane protein types [20, 22]. To extract rich evolutionary information from PSSM, many researchers have proposed their own methods, such as the reduced position-specific score matrix (RPSSM) [23], evolutionary difference position-specific score matrix (EDPSSM) [24], tri-gram position-specific score matrix (TriPSSM) [25], k-separated-bigrams position-specific score matrix (KPSSM) [26], correlation position-specific scoring matrix (CoPSSM) [27], and pseudo position-specific score matrix (PsePSSM) [9]. These methods are all obtained through complex feature engineering.

In this paper, we improve the performance in membrane protein type prediction. The main contributions of the paper are summarized as follows: First, we present two deep neural network (DNN) models to process the sequence information and evolutionary information separately. These models both achieve better performance than traditional machine learning models. Second, by using the DNN model with convolutional and recurrent layers, we can remove the burden of feature engineering and the reliance on domain experts. Third, we provide a new vector representation based on autoencoder and physicochemical property indexes. Numerous experimental results prove that the new vector has more powerful representation ability than one-hot encoding. Finally, a more effective model is constructed by fusing the above two models with the ensemble learning.

The DNN model has shown its superiority in the field of bioinformatics [28-37]. However, to the best of our knowledge, this paper is the first to propose DNN models for the prediction of membrane protein types. The two DNN models proposed in this paper can process the sequence information and evolutionary information, separately. When processing sequence information, we use a 1D convolutional layer [38] and bidirectional long short term-memory (Bi-LSTM) layers [39], and when processing evolutionary information, a 2D convolutional layer and CapsNet layer [40] are adopted. Among these layers, compared to convolutional network, CapsNet is a new network that can extract local position information.

\section{Methods}

\section{Datasets}

In this work, we use two datasets to evaluate the performance of our method, hereafter referred to as Dataset 1 and Dataset 2. Dataset 1 consists of two benchmark sets, the training set and the testing set, which are both from paper [41] and used in previous studies [9, 10, 12, 13, 20]. Dataset 2 is constructed by Chen [12]; it also consists of two benchmark sets. Table 1 shows the concrete distribution of Dataset 1 and Dataset 2.

\section{Architecture of the proposed DNN}

To improve the performance of the prediction of membrane protein types, we build the DNN model with a keras framework (http://www.keras.io). Considering the respective characteristics of sequence information and evolutionary information, we build two different models to process them, which are hereafter referred to as the sequence information model and evolutionary information model, respectively.

\section{Sequence information model}

In this model, the input sequences are converted into numerical vectors of fixed length by truncating the long sequence and padding the short sequence with ' 0 '. The length of the sequence ranges from 50 to 5000 in our dataset. Although the length of the sequence varies 
Table 1 The distribution of samples for Dataset 1 and Dataset 2

\begin{tabular}{|c|c|c|c|c|}
\hline \multirow[t]{2}{*}{ Membrane protein types } & \multicolumn{2}{|l|}{ Dataset 1} & \multicolumn{2}{|l|}{ Dataset 2} \\
\hline & Training set & Testing set & Training set & Testing set \\
\hline Type I & 610 & 444 & 561 & 245 \\
\hline Type II & 312 & 78 & 316 & 79 \\
\hline Type III & 24 & 6 & 32 & 9 \\
\hline Type IV & 44 & 12 & 65 & 17 \\
\hline Mutipass & 1316 & 3265 & 1119 & 2478 \\
\hline Lipid-chain-anchored & 151 & 38 & 142 & 36 \\
\hline GPI-anchored & 182 & 46 & 164 & 41 \\
\hline Peripheral & 610 & 444 & 674 & 699 \\
\hline overall & 3249 & 4333 & 3073 & 3604 \\
\hline
\end{tabular}

greatly, sequences with a length of less than 1500 account for almost $98 \%$ of the total dataset. Therefore, taking the utilization of information and the computational complexity into account, we decided to set the fixed length to 1500. Each of the basic 20 amino acids is converted into a number of 0-19. Ultimately, to prevent different encoding from affecting the performance of model, one-hot encoding is used before inputting the vector into the model.

The architecture of the sequence information model, as shown in Fig. 1, consists of one 1D convolutional layer (filters: 256, kernel_size: 15, strides: 10, padding: 'same', activation: 'relu'), two Bi-LSTM layers (units: 128, dropout: 0.5 and rest default setting) and one fully connected layer (softmax). The 1D convolutional layer can reduce the complexity of the Bi-LSTM layer that reduces the shape of the output matrix from $1500 \times 256$ tensor to $150 \times 256$. Moreover, the 256 filters in the layer can also extract rich information from the sequence. Next, two Bi-LSTM layers are applied, which can identify features separated by large gaps. In previous studies, a normal LSTM layer is mainly used to process sequence information $[29,30]$. Compared to normal LSTM, the Bi-LSTM

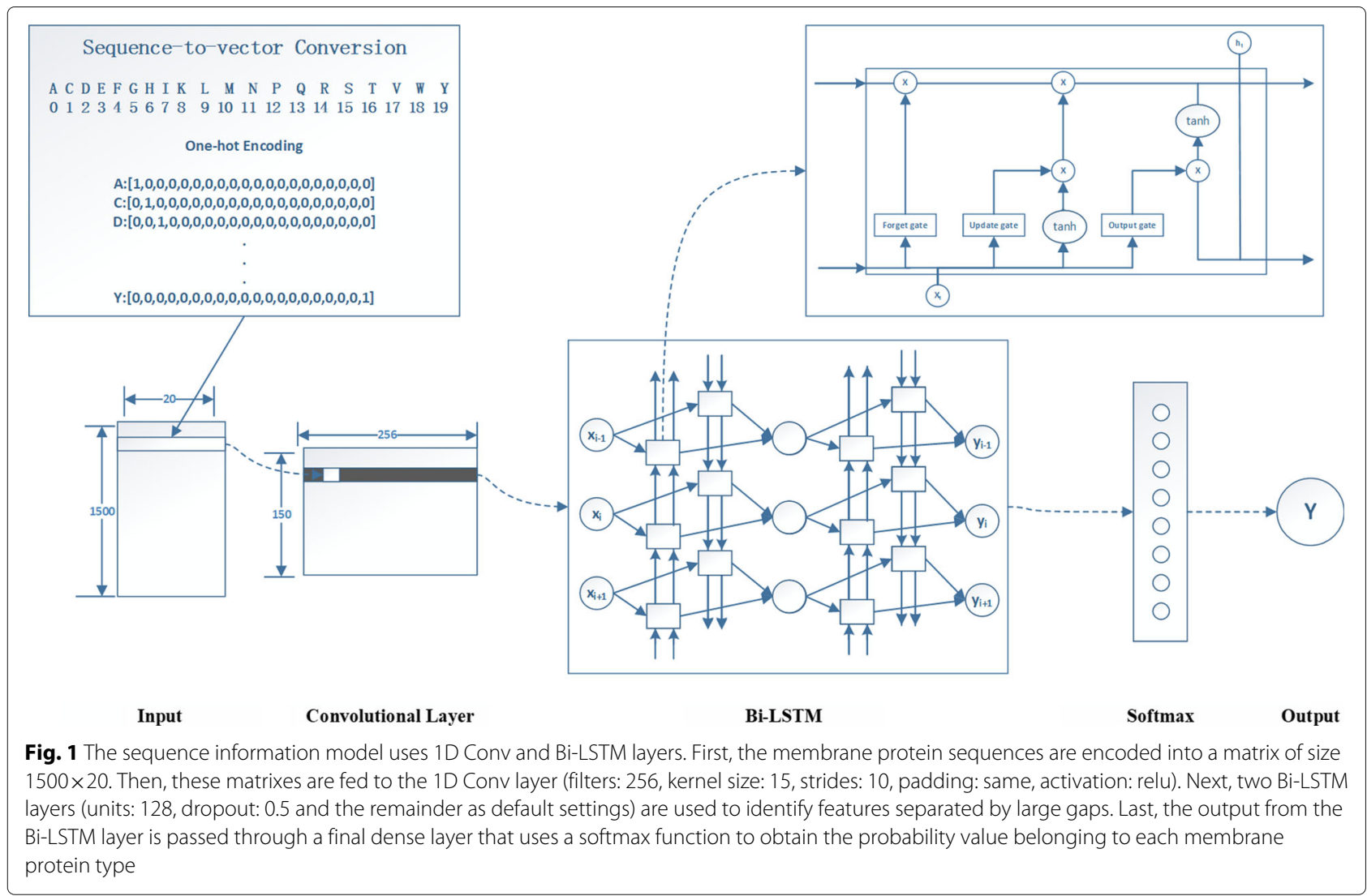


layer we used can take advantage of both historical input and future information, which performs better for our task. Each Bi-LSTM unit comprises three gates, which are shown in Fig. 1. Their calculation process is as follows:

$$
\begin{aligned}
& f_{t}=\sigma\left(W_{f} \cdot\left[h_{t-1}, x_{t}\right]+b_{f}\right) \quad \text { forget gate } \\
& i_{t}=\sigma\left(W_{i} \cdot\left[h_{t-1}, x_{t}\right]+b_{i}\right) \quad \text { input gate } \\
& \tilde{C}_{t}=\tanh \left(W_{C} \cdot\left[h_{t-1}, x_{t}\right]+b_{C}\right) \quad \text { candidate } \\
& C_{t}=f_{t} * C_{t-1}+i_{t} * \tilde{C}_{t} \quad \text { updated cell states } \\
& o_{t}=\sigma\left(W_{o} \cdot\left[h_{t-1}, x_{t}\right]+b_{o}\right) \quad \text { output gate } \\
& \left.h_{t}=o_{t} * \tanh \left(C_{t}\right)\right) \quad \text { hidden cell states }
\end{aligned}
$$$$
\text { candidate cell states }
$$

where $L$ is the length of the sequence, and $M_{i \rightarrow j}$ denotes the score of the amino acid in the $i$-th position of the amino acid being mutated to $j$-th position of the amino acid during the evolution process. Because membrane protein sequences vary in size, their PSSM shapes are also different. Similar to the sequence information model, we also truncate the long sequence and pad the short sequence with ' 0 ' to convert the PSSM matrix into a $1500 \times 20$ matrix

The architecture of evolutionary information model is shown in Fig. 2. It consists of two 2D convolutional layers, two average pooling layers, one PrimaryCaps and one fully connected layer. The first 2D convolutional layer (filters: 256, kernel_size: $(5,5)$, strides: 1 , padding: 'valid', activation: 'relu') is used to extract rich and effective features from the PSSM. Next, the first 2D average pooling layer (pool_size: $(2,2)$ and the remainder as default settings) is used to retain the main features while reducing the parameters and calculations of the next layer to prevent overfitting. Although paper [40] argued that the pooling layer may throw away information about the precise position of the entity within the region, we find that the pooling layer and CapsNet work together to obtain the best results in our task. One possible reason is that the pooling layer can improve the robustness of extracted features. Then, the second 2D convolutional layers (filters: 128, kernel_size: $(5,5)$, strides: 1, padding: 'valid', activation: 'relu') and the second $2 \mathrm{D}$ average pooling layer (pool_size: $(2,2)$ and the remainder as default settings) are reused to obtain more advanced features. Moreover, each convolutional layer has a dropout technique with the rate 0.5 to prevent the model from overfitting. The first four layers are designed to increase the representation power of CapsNet.

The PrimaryCaps layer contains 4 primary capsules that accept the basic features detected by the first four layers and generate a combination of features. The 4 main capsules of this layer are essentially similar to the convolutional layer. Among them, the $1 \times 1$ convolution kernel used in our study not only achieves cross-channel interaction and information integration, but also reduces the number of convolution kernel channels. Each capsule applies sixteen $1 \times 1 \times 64$ convolution kernels to the $372 \times 2 \times 128$ input tensor, thus generating a $372 \times 2 \times 64$ output tensor. With a total of 4 capsules, the output is a $372 \times 2 \times 4 \times 16$ tensor. Since the length of a capsule

\section{Evolutionary information model}

In this paper, evolutionary information mainly refers to the PSSM, which was first proposed by Jones for predicting the secondary structure of proteins [42]. The PSSM can discover protein sequences that have evolved relation-

$$
P S S M=\left[\begin{array}{cccc}
M_{1 \rightarrow 1} & M_{1 \rightarrow 2} & \cdots & M_{1 \rightarrow 20} \\
M_{2 \rightarrow 1} & M_{2 \rightarrow 2} & \cdots & M_{2 \rightarrow 20} \\
\vdots & \vdots & \ddots & \vdots \\
M_{L \rightarrow 1} & M_{L \rightarrow 2} & \cdots & M_{L \rightarrow 20}
\end{array}\right]
$$




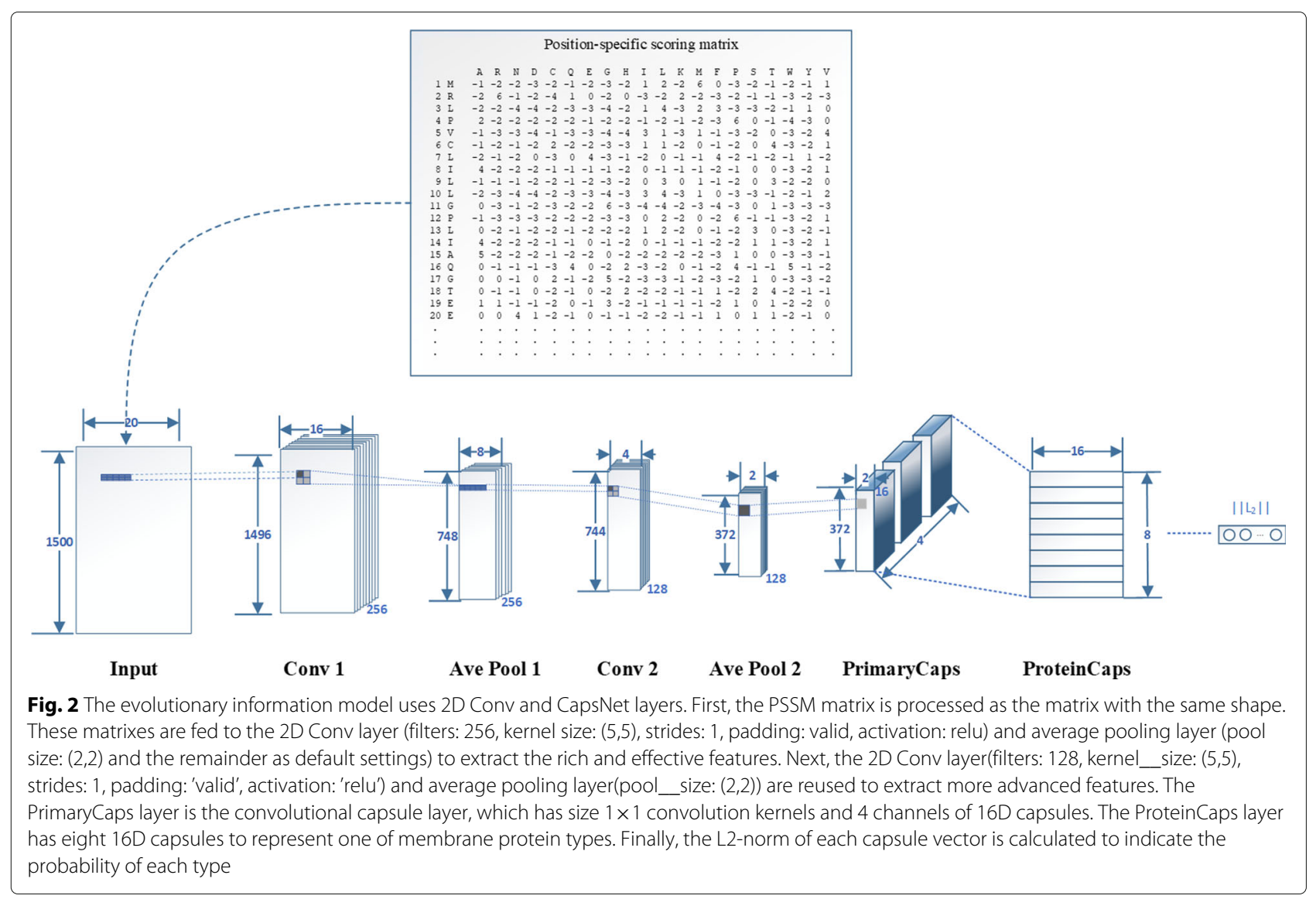

represents the probability that the entity presented, the CapsNet layer needs a new activation function, which is called the squashing function. It is a novel nonlinear activation function that accepts an input vector and then compresses its length to $[0,1]$ as output, which is calculated as follows:

$$
V_{j}=\frac{\left\|s_{j}\right\|}{1+\left\|s_{j}\right\|^{2}} \frac{s_{j}}{\left\|s_{j}\right\|}
$$

where $V_{j}$ and $s_{j}$ are the vector output and input of the CapsNet, respectively.

The last layer contains eight membrane protein type capsules, one for each type of membrane protein. The calculation between the PrimaryCaps layer and ProteinCaps layer is shown in Fig. 3. Among them, $u_{i}$ represents the $i$ th capsule in PrimaryCaps. $W_{i j}$ is the weight matrix that represents the spatial relationship and other important relationships between low-level capsules and high-level capsules. There are eight capsules $\left(V_{j}, j \in[1,2, \ldots, 8]\right)$ in ProteinCaps, each of which receives the inputs from all capsule outputs in PrimaryCaps. $V_{j}$ is calculated by the weighted sum of $\tilde{u}_{j i}$ and then is passed through the squashing function. Here, the weights $c_{i j}$ are obtained by an iterative dynamic routing process that can be found in [40].
The output of the ProteinCaps layer has eight $16 \mathrm{D}$ vectors. During training, for each training sample, the loss value is calculated as follows:

$$
\left.\left.L_{c}=T_{c} \max \left(0, m^{+}-\left\|V_{c}\right\|\right)\right)^{2}+\lambda\left(1-T_{c}\right) \max \left(0,\left\|V_{c}\right\|\right)-m^{-}\right)^{2}
$$

where the value of $T_{c}$ is decided by the correct label. If the correct label corresponds to the membrane protein type in ProteinCaps, then $T_{c}=1$; otherwise, $T_{c}=0$. For the value of the other hyperparameters $m^{+}, m^{-}$and $\lambda$, we used the default values $0.9,0.1$ and 0.5 , respectively, which are suggested in [40].Then, eight loss values are added to obtain the final loss.

Finally, we use the 'Adam' optimizer method (lr: 0.0001, beta_1: 0.9, beta_2: 0.999, decay=0). The suggested settings, three routing iterations and the margin loss function, are used in our work.

\section{Fusion information model}

Many studies have proved that the fusing information method has better results than the single information method [12, 20, 43-45]. Therefore, we also present a fusion information model based on ensemble learning. Among these methods, the ensemble learning method 


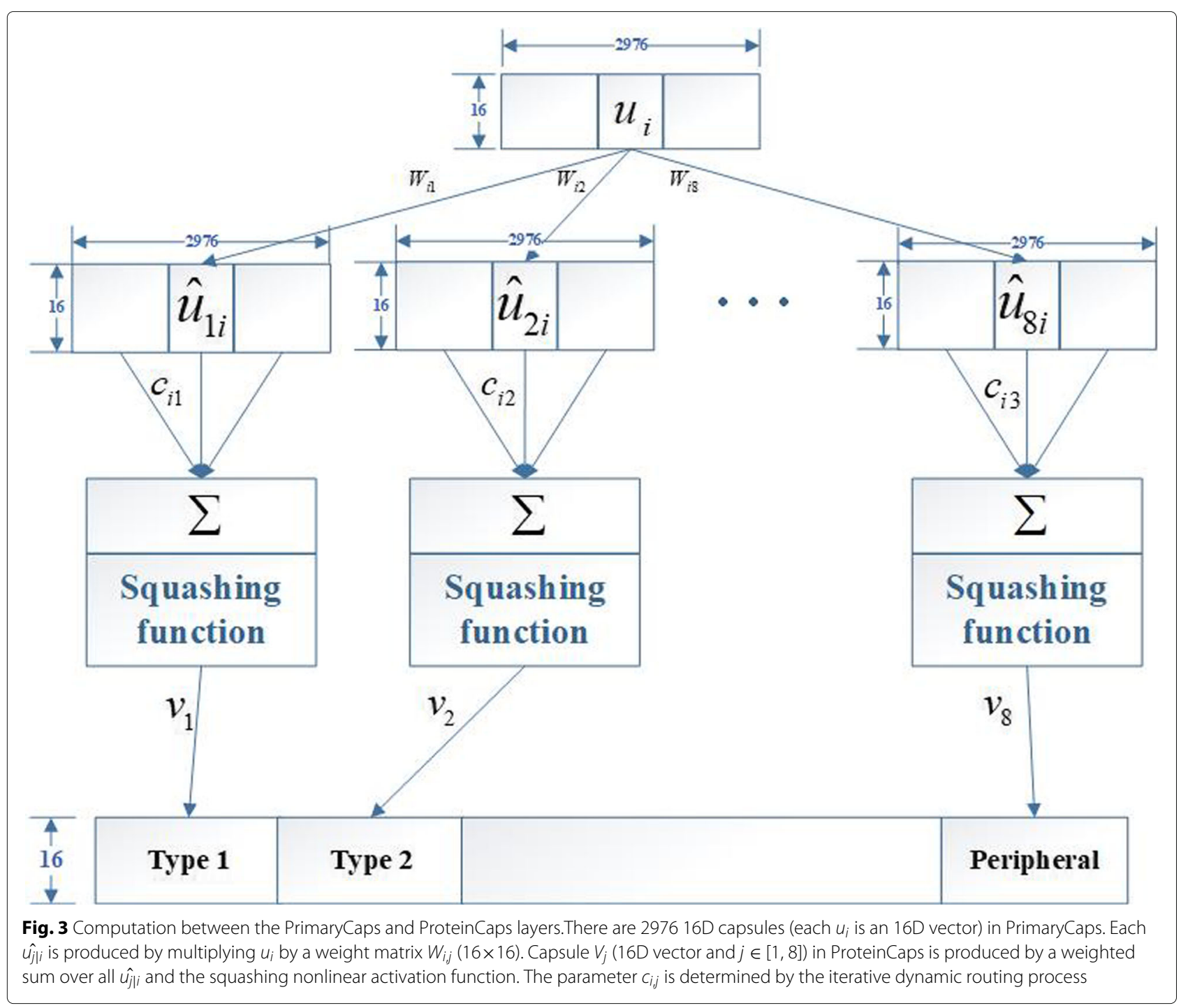

that we adopted is a stacking algorithm, and its details can be found in our previous work [19]. Figure 4 shows the complete flow chart of the stacking algorithm, where the meta-classifier that we used is multiple logistic regression (MLR).

Research has shown that using the class probability output of the base classifier as the input of the meta classifier performs better [46]. Then, we use $P(x)$ as the output of the base classifier, it can be represented as follows:

$$
\begin{aligned}
P(x) & =\left(P_{1}^{\mathrm{T}}, P_{2}^{\mathrm{T}}\right) \\
& =(\underbrace{P_{1}^{1}, P_{2}^{1}, P_{3}^{1}, P_{4}^{1}, P_{5}^{1}, P_{6}^{1}, P_{7}^{1}, P_{8}^{1}}_{\text {Sequence_informtaion_model }}, \underbrace{P_{1}^{2}, P_{2}^{2}, P_{3}^{2}, P_{4}^{2}, P_{5}^{2}, P_{6}^{2}, P_{7}^{2}, P_{8}^{2}}_{\text {Evolutionary_informtaion_model }})
\end{aligned}
$$

\section{Model training}

In most experiments, the DNN models are trained using identical training strategies. The dataset is divided into three parts, the training set, validation set and testing set, which play different roles in our work. The training set is used to train the model, and the validation set is used to adjust parameters and select the best model. The testing set is used to evaluate the performance of the model at the end. In our work, $20 \%$ of samples are separated from the original training set as a validation set. Then, the distributions of three sets in two datasets are shown in Table 2. All DNN models are implemented using keras 2.1.2. Model training and testing are performed on a personal computer equipped with an Nvidia GTX 1060 GPU.

\section{Model evaluation}

We use sensitivity (Se), specificity (Sp), accuracy (ACC) and overall success rate (OSR) to evaluate the classification performance. They are defined as follows:

$$
S e_{i}=T P_{i} /\left(T P_{i}+F N_{i}\right)
$$




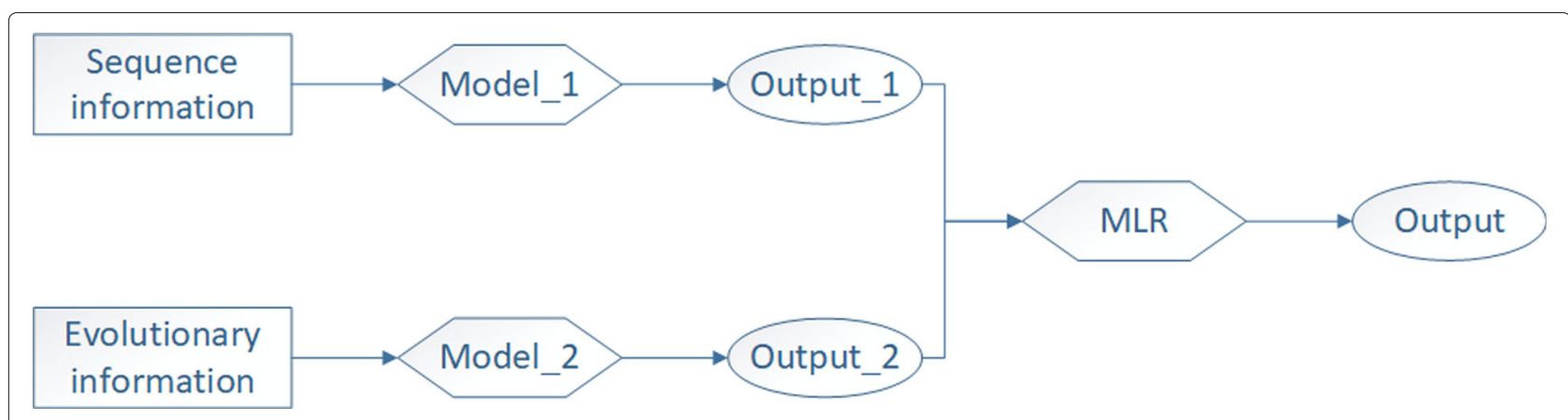

Fig. 4 The flow of the stacking algorithm. Model_1 refers to the sequence information model, and Model_2 refers to the evolutionary information model. The class probability outputs of two models are combined to serve as a new training set to train the meta-classifier (MLR). When testing unknown membrane protein samples, we take the result of the meta-classifier as the final output

$$
\begin{aligned}
& S p_{i}=T N_{i} /\left(T N_{i}+F P_{i}\right) \\
& A C C_{i}=\frac{T P_{i}+T N_{i}}{T P_{i}+T N_{i}+F N_{i}+F P_{i}} \\
& O S R=\frac{\sum_{i=1}^{k} T P_{i}}{\sum_{i=1}^{k}\left(T P_{i}+F N_{i}\right)}
\end{aligned}
$$

where $T P, T N, F P$ and $F N$ represent true positive, true negative, false positive and false negative, respectively.

The Matthews correlation coefficient (MCC) is also used in our work. It is generally considered to be a more balanced indicator, even if the sample content of the two categories varies widely [47]. The MCC is essentially a correlation coefficient between the actual classification and the predicted classification. Its value range is $[-1,1]$. A value of 1 indicates a perfect prediction for the subject. A value of 0 indicates that the predicted result is not as good as the result of random prediction, and -1 means that the predicted classification is completely inconsistent with the actual classification. The MCC is defined as follows:

$$
M C C_{i}=\frac{T P_{i} \times T N_{i}-F P_{i} \times F N_{i}}{\sqrt{\left(T P_{i}+F N_{i}\right)\left(T P_{i}+F P_{i}\right)\left(T N_{i}+F P_{i}\right)\left(T N_{i}+F N_{i}\right)}}
$$

\section{The construction of vector representation}

One-hot encoding ignores the possible relationship between amino acids. For example, alanine and cysteine have a certain relationship in their physicochemical properties, yet ' 0 ' and ' 1 ' do not provide much informtaion about this relationship. Moreover, we usually need more data to train because the amino acids are stored as sparse matrixes. Using a vector representation method can effectively solve the above problems. Continuous bag of words (CBOW) and Skim-Gram [48] are two popular vector representation methods that are widely used in the field of natural language processing (NLP). Limited by the size of protein samples and the characteristics of the protein sequence, these methods are no longer applicable. In this

\begin{tabular}{|c|c|c|c|c|c|c|}
\hline \multirow{2}{*}{ Membrane protein types } & \multicolumn{3}{|l|}{ Dataset 1} & \multicolumn{3}{|l|}{ Dataset 2} \\
\hline & Training set & Validation set & Testing set & Training set & Validation set & Testing set \\
\hline Type I & 488 & 122 & 444 & 449 & 112 & 245 \\
\hline Type II & 250 & 62 & 78 & 253 & 63 & 79 \\
\hline Type III & 19 & 5 & 6 & 25 & 7 & 9 \\
\hline Type IV & 35 & 9 & 12 & 52 & 13 & 17 \\
\hline Mutipass & 1052 & 264 & 3265 & 895 & 224 & 2478 \\
\hline Lipid-chain-anchored & 121 & 30 & 38 & 114 & 28 & 36 \\
\hline GPI-anchored & 146 & 36 & 46 & 131 & 33 & 41 \\
\hline Peripheral & 488 & 122 & 444 & 539 & 135 & 699 \\
\hline overall & 2599 & 650 & 4333 & 2458 & 615 & 3604 \\
\hline
\end{tabular}
paper, we provide a new vector representation method based on autoencoder and the physicochemical properties

Table 2 The distribution of three data in two datasets 
of amino acids. This method can achieve a more compact representation of input symbols and yield semantically similar symbols close to each other in vector space.

The AAindex is a database of numerical indexes representing various physicochemical properties of amino acids and pairs of amino acids [49]. The database now has a total of 557 indexes, which all come from the published paper. In this research, the physicochemical properties indexes with ' $\mathrm{NA}^{\prime}$ are screened out, and the remaining 537 indexes are adopted. Using a physicochemical properties index that represents the amino acids is a good idea to replace the one-hot encoding. However, this method would consume a large amount of storage space and greatly increase the computational complexity. To process the above problems, autoencoder is used to create a more effective representation.

Autoencoder is a neural network that can capture the most important features of data. It consists of the input layer, multiple hidden layers and output layer. The input and output of the autoencoder are consistent, and the goal is to reconstruct itself using some high-level feature recombination. If the number of nodes of the hidden layer is smaller than the input layer and the output layer, it represents the same low-density information and is a centralized representation of the input data obtained from the learning. In our work, we take 537 physicochemical properties indexes of each amino acid as input, reconstruct this informatin with the autoencoder, and then obtain a more effective vector representation from the intermediate layer. The architecture of the autoencoder that we used is shown in Fig. 5.

\section{Results and discussion}

The performance of vector representation

To explore the advantage of the new vector representation method, we compared the performance between the onehot encoding and our method in the sequence information model. We note that our process of parameter adjustment is all on the validation set and not reparameterized to apply on the testing set.

Figure 6 shows the overall success rate of vector representations using different dimensions in Dataset 1 and Dataset 2 . To verify whether the vector representation that we proposed is better than one-hot encoding, we also indicated the overall success rate of the one-hot encoding method in the figures. It can be seen from the figure that our method is better in most cases. Taking both the prediction performance and computational complexity into account, we finally chose the vector representation with 10-dimension to replace the one-hot encoding.

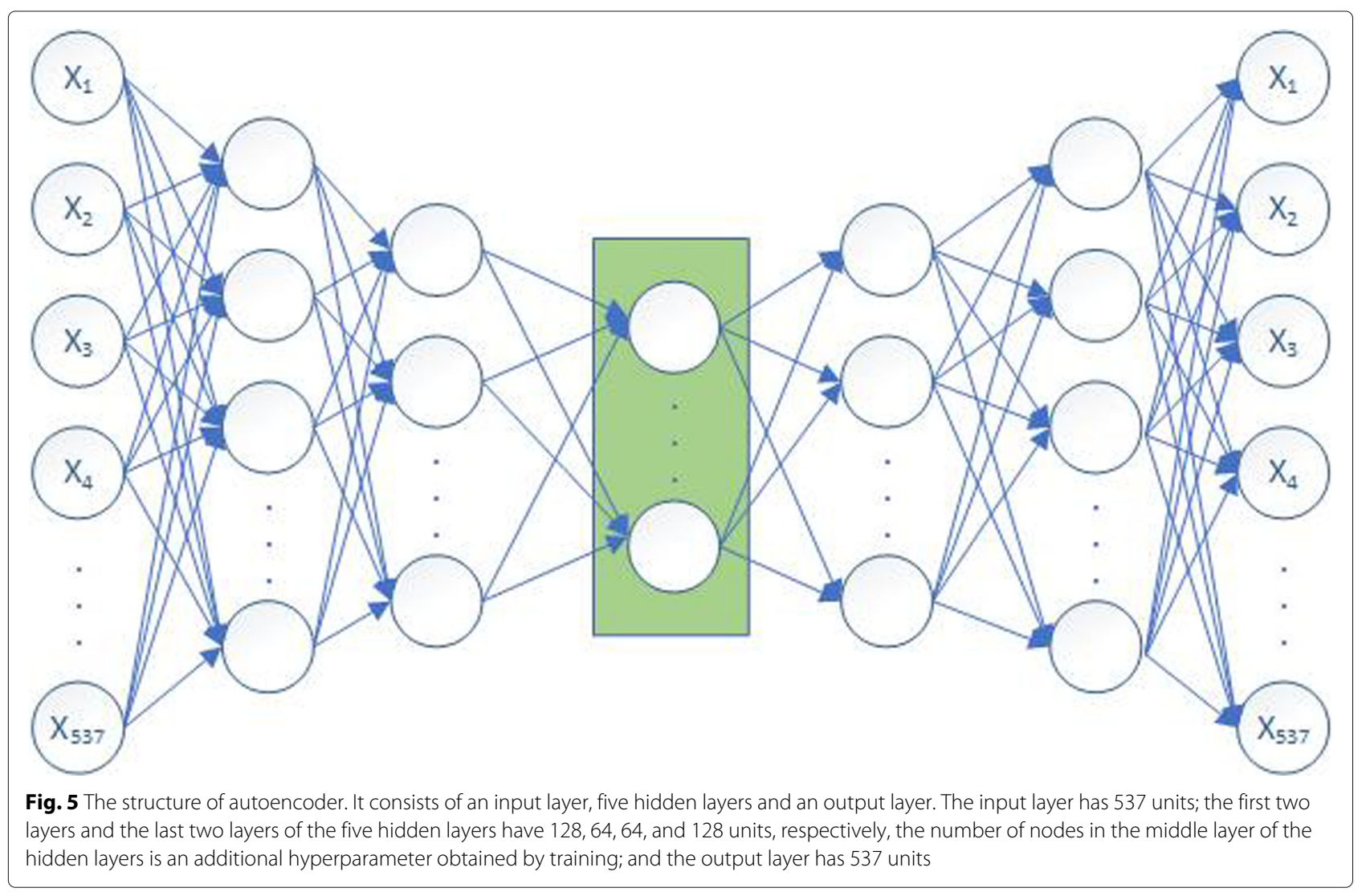




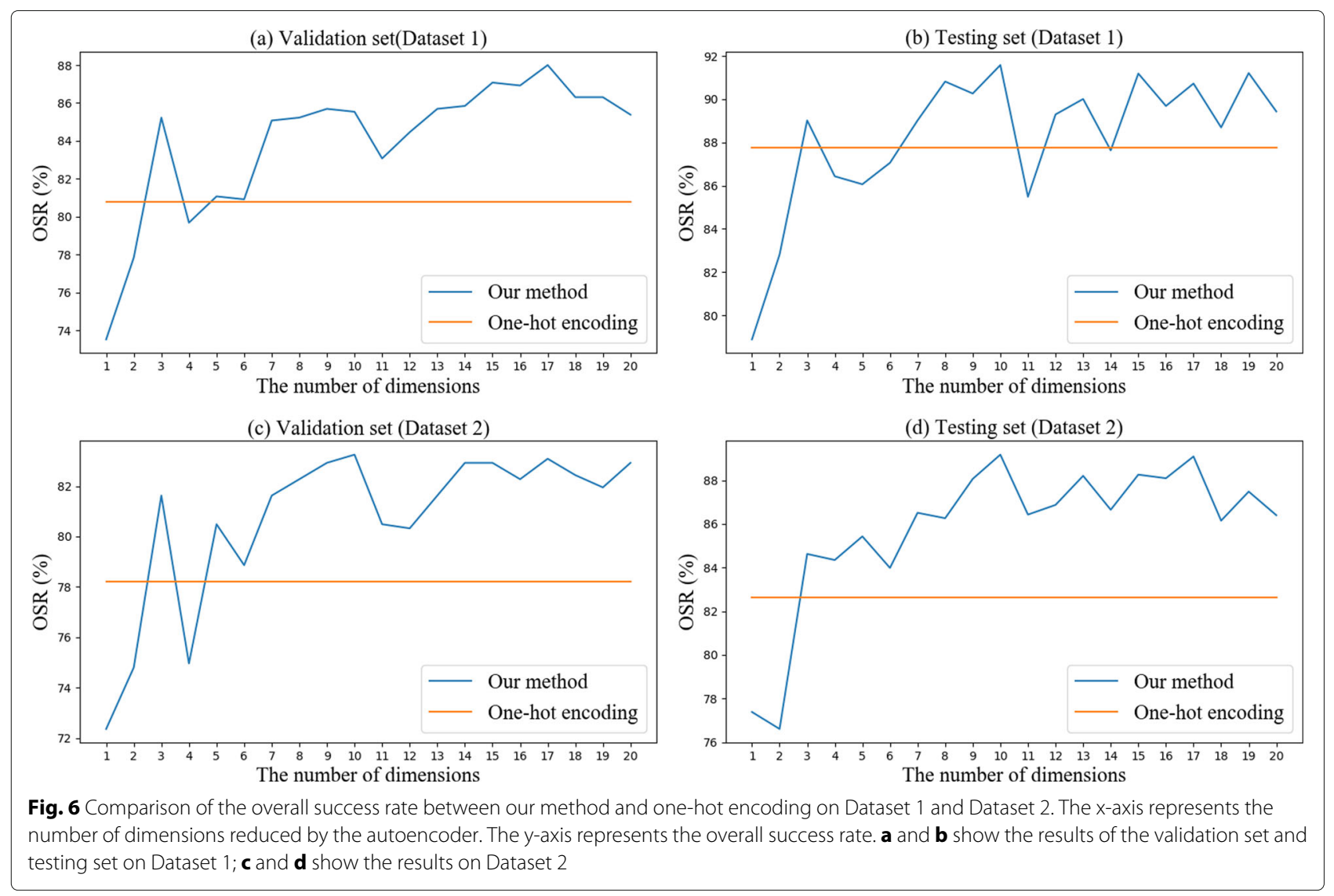

Furthermore, to verify whether the vector representation is helpful to improve the performance of the model, we also randomly generate a 10-dimensional vector in the interval $[0,1]$ for comparison experiments; the results are shown in Table 3. From the table, we find that the vector representation that we proposed is more effective than other methods both on Dataset 1 and Dataset 2. In addition, we find that the random method is even worse than the one-hot encoding. Although the random method has a lower dimension, it cannot express the relationship between amino acids well, which makes the result worse. The final experimental results illustrate the reliability of the new vector representation method that we proposed.

Table 3 Comparison of the overall success rate between different vector representation methods on Dataset 1 and Dataset 2

\begin{tabular}{|c|c|c|c|c|}
\hline \multirow{2}{*}{ Method } & \multicolumn{2}{|l|}{ Dataset 1} & \multicolumn{2}{|l|}{ Dataset 2} \\
\hline & Validation set & Testing set & Validation set & Testing set \\
\hline One hot encoding & $80.78 \%$ & $87.77 \%$ & $78.21 \%$ & $82.63 \%$ \\
\hline Random method & $74.77 \%$ & $80.87 \%$ & $71.22 \%$ & $81.94 \%$ \\
\hline Our method & $85.54 \%$ & $91.58 \%$ & $83.25 \%$ & $89.18 \%$ \\
\hline
\end{tabular}

Note: Best performing method in bold.

\section{The effective of sequence information model}

Table 4 gives the overall success rate of the comparison between the sequence information model and traditional models on Dataset 1 and Dataset 2, where four popular features used for comparative experiments are AAC, DipC, TipC and PseAAC. Among them, AAC, DipC and Tipc are amino acid composition-based methods; PseAAC is the method based on amino acid composition and physicochemical properties. These features are all extracted from the membrane protein sequence. The machine learning algorithm used to compare random

Table 4 Comparison of the overall success rate between four traditional models and sequence information model on Dataset 1 and Dataset 2

\begin{tabular}{llll}
\hline \multirow{2}{*}{ Method } & Dataset 1 & & Dataset 2 \\
\cline { 2 - 2 } & Overall success rate & & Overall success rate \\
\hline AAC+RF & $85.02 \%$ & $79.55 \%$ \\
DipC+RF & $86.12 \%$ & $80.88 \%$ \\
TipC+RF & $84.21 \%$ & $79.00 \%$ \\
PseAAC+RF & $85.74 \%$ & $80.27 \%$ \\
Our model & $\mathbf{9 1 . 5 8} \%$ & $\mathbf{8 9 . 1 8 \%}$ \\
\hline
\end{tabular}

Note: Best performing method in bold. 
forests. Research has illustrated its effectiveness in the prediction of membrane protein types [50]. The optimal parameters in random forests are obtained by using the grid search method and 5-fold cross-validation on the training set. From the table, we find that our DNN model has a better overall success rate than all the machine learning methods. Compared with the $\mathrm{DipC}$, which has the best results among the four sequence features, our model improved by $5.43 \%$ and $8.30 \%$ on Dataset 1 and Dataset 2, respectively.

Tables 5 and 6 give the values of Se, Sp, ACC and MCC for eight membrane protein types using four traditional models and our DNN model on Dataset 1 and Dataset 2 , respectively. Although our model is not as good as the traditional model in some indicators, our model achieves better results in most cases. Furthermore, we find that it

Table 5 Comparison of the Se, Sp, ACC and MCC between four traditional models and sequence information model on Dataset 1

\begin{tabular}{|c|c|c|c|c|c|c|}
\hline Membrane protein types & Index & AAC & DipC & TipC & PseAAC & Our model \\
\hline & Se & 0.7928 & 0.7095 & 0.5225 & 0.8221 & 0.8356 \\
\hline \multirow[t]{4}{*}{ Type I } & $\mathrm{Sp}$ & 0.9537 & 0.9671 & 0.9805 & 0.9568 & 0.9941 \\
\hline & ACC & 0.9372 & 0.9407 & 0.9335 & 0.9430 & 0.9778 \\
\hline & MCC & 0.6898 & 0.6772 & 0.5936 & 0.7192 & 0.8751 \\
\hline & Se & 0.2692 & 0.0641 & 0.0256 & 0.2949 & 0.8077 \\
\hline \multirow[t]{4}{*}{ Type II } & Sp & 0.9946 & 0.9993 & 1.0 & 0.9951 & 0.9871 \\
\hline & ACC & 0.9815 & 0.9825 & 0.9825 & 0.9825 & 0.9838 \\
\hline & MCC & 0.3499 & 0.1964 & 0.1587 & 0.3845 & 0.6492 \\
\hline & $\mathrm{Se}$ & 0.0 & 0.0 & 0.0 & 0.0 & 0.1667 \\
\hline \multirow[t]{4}{*}{ Type III } & Sp & 1.0 & 1.0 & 1.0 & 1.0 & 0.9998 \\
\hline & ACC & 0.9986 & 0.9986 & 0.9986 & 0.9986 & 0.9986 \\
\hline & MCC & 0.0 & 0.0 & 0.0 & 0.0 & 0.2881 \\
\hline & $\mathrm{Se}$ & 0.0833 & 0.0 & 0.0 & 0.0 & 0.6667 \\
\hline \multirow[t]{4}{*}{ Type IV } & $S p$ & 1.0 & 1.0 & 1.0 & 1.0 & 0.9984 \\
\hline & ACC & 0.9975 & 0.9972 & 0.9972 & 0.9972 & 0.9986 \\
\hline & MCC & 0.2883 & 0.0 & 0.0 & 0.0 & 0.5950 \\
\hline & $\mathrm{Se}$ & 0.9069 & 0.9587 & 0.9816 & 0.9029 & 0.9461 \\
\hline \multirow[t]{4}{*}{ Mutipass } & $S p$ & 0.8661 & 0.7004 & 0.4766 & 0.8858 & 0.9092 \\
\hline & ACC & 0.8968 & 0.8950 & 0.8571 & 0.8987 & 0.9370 \\
\hline & MCC & 0.7390 & 0.7051 & 0.5847 & 0.7479 & 0.8355 \\
\hline & $\mathrm{Se}$ & 0.1579 & 0.0789 & 0.0263 & 0.1579 & 0.1579 \\
\hline \multirow[t]{4}{*}{ Lipid-chain-anchored } & $S p$ & 0.9988 & 1.0 & 1.0 & 0.9993 & 0.9979 \\
\hline & ACC & 0.9915 & 0.9919 & 0.9915 & 0.9919 & 0.9905 \\
\hline & MCC & 0.2904 & 0.2798 & 0.1615 & 0.3219 & 0.2473 \\
\hline & $\mathrm{Se}$ & 0.3696 & 0.0435 & 0.0435 & 0.3478 & 0.8478 \\
\hline \multirow[t]{4}{*}{ GPI-anchored } & Sp & 0.9974 & 1.0 & 1.0 & 0.9960 & 0.9951 \\
\hline & ACC & 0.9908 & 0.9898 & 0.9898 & 0.9892 & 0.9935 \\
\hline & MCC & 0.4694 & 0.2075 & 0.2075 & 0.4054 & 0.7393 \\
\hline & $\mathrm{Se}$ & 0.7342 & 0.6261 & 0.4662 & 0.8041 & 0.8806 \\
\hline \multirow[t]{3}{*}{ Peripheral } & $\mathrm{Sp}$ & 0.9262 & 0.9617 & 0.9874 & 0.9262 & 0.9609 \\
\hline & ACC & 0.9065 & 0.9273 & 0.9340 & 0.9137 & 0.9527 \\
\hline & MCC & 0.5747 & 0.5981 & 0.5835 & 0.6226 & 0.7708 \\
\hline
\end{tabular}


Table 6 Comparison of the Se, Sp, ACC and MCC between four traditional models and sequence information model on Dataset 2

\begin{tabular}{|c|c|c|c|c|c|c|}
\hline Membrane protein types & Index & AAC & DipC & TipC & PseAAC & Our model \\
\hline & Se & 0.6571 & 0.5306 & 0.3388 & 0.6898 & 0.8408 \\
\hline \multirow[t]{4}{*}{ Type I } & $\mathrm{Sp}$ & 0.9384 & 0.9643 & 0.9824 & 0.9440 & 0.9806 \\
\hline & ACC & 0.9193 & 0.9348 & 0.9387 & 0.9267 & 0.9711 \\
\hline & MCC & 0.4951 & 0.4903 & 0.4156 & 0.5341 & 0.7841 \\
\hline & Se & 0.1139 & 0.0 & 0.0 & 0.0633 & 0.7468 \\
\hline \multirow[t]{4}{*}{ Type II } & $\mathrm{Sp}$ & 0.9901 & 1.0 & 1.0 & 0.9889 & 0.9739 \\
\hline & ACC & 0.9709 & 0.9781 & 0.9781 & 0.9686 & 0.9689 \\
\hline & MCC & 0.1387 & 0.0 & 0.0 & 0.0696 & 0.5267 \\
\hline & Se & 0.0 & 0.0 & 0.0 & 0.0 & 0.0 \\
\hline \multirow[t]{4}{*}{ Type III } & $\mathrm{Sp}$ & 1.0 & 1.0 & 1.0 & 1.0 & 1.0 \\
\hline & ACC & 0.9975 & 0.9975 & 0.9975 & 0.9975 & 0.9975 \\
\hline & MCC & 0.0 & 0.0 & 0.0 & 0.0 & 0.0 \\
\hline & Se & 0.0 & 0.0 & 0.0 & 0.0 & 0.7647 \\
\hline \multirow[t]{4}{*}{ Type IV } & $\mathrm{Sp}$ & 1.0 & 1.0 & 1.0 & 1.0 & 0.9958 \\
\hline & ACC & 0.9953 & 0.9953 & 0.9953 & 0.9953 & 0.9947 \\
\hline & MCC & 0.0 & 0.0 & 0.0 & 0.0 & 0.5935 \\
\hline & Se & 0.8721 & 0.9290 & 0.9617 & 0.8709 & 0.9157 \\
\hline \multirow[t]{4}{*}{ Mutipass } & $\mathrm{Sp}$ & 0.8526 & 0.7105 & 0.4885 & 0.8588 & 0.9813 \\
\hline & ACC & 0.8660 & 0.8607 & 0.8138 & 0.8671 & 0.9362 \\
\hline & MCC & 0.7022 & 0.6669 & 0.5442 & 0.7058 & 0.8638 \\
\hline & Se & 0.0556 & 0.0 & 0.0 & 0.0278 & 0.0 \\
\hline \multirow[t]{4}{*}{ Lipid-chain-anchored } & $\mathrm{Sp}$ & 1.0 & 1.0 & 1.0 & 1.0 & 0.9983 \\
\hline & ACC & 0.9906 & 0.9900 & 0.9900 & 0.9903 & 0.9883 \\
\hline & MCC & 0.2346 & 0.0 & 0.0 & 0.1659 & -0.0041 \\
\hline & $\mathrm{Se}$ & 0.3659 & 0.0488 & 0.0 & 0.3659 & 0.9024 \\
\hline \multirow[t]{4}{*}{ GPI-anchored } & $\mathrm{Sp}$ & 0.9986 & 1.0 & 1.0 & 0.9941 & 0.9938 \\
\hline & ACC & 0.9914 & 0.9892 & 0.9886 & 0.9870 & 0.9928 \\
\hline & MCC & 0.5203 & 0.2197 & 0.0 & 0.3839 & 0.7490 \\
\hline & $\mathrm{Se}$ & 0.7425 & 0.6881 & 0.5451 & 0.7797 & 0.9013 \\
\hline \multirow[t]{3}{*}{ Peripheral } & $\mathrm{Sp}$ & 0.8885 & 0.9164 & 0.9580 & 0.8954 & 0.9418 \\
\hline & ACC & 0.8602 & 0.8721 & 0.8779 & 0.8729 & 0.9340 \\
\hline & MCC & 0.5893 & 0.5965 & 0.5740 & 0.6290 & 0.8025 \\
\hline
\end{tabular}

Note: Best performing method in bold.

is difficult for the method combining feature engineering and the machine learning algorithm to predict the small types, especially in Type 4 membrane protein samples where the value of $\mathrm{Sn}$ and Mcc is zero in most traditional models. However, our model can predict the small type samples well. The reason may be the powerful feature extraction capabilities of the convolutional layer and $\mathrm{Bi}$ LSTM layer that we used. The experimental results prove that our proposed sequence information model has better performance than traditional models.

\section{The effective of evolutionary information model}

Then, we use the same experimental method to compare the performance between the evolutionary information model and seven popular PSSM-based tradition methods proposed by other researchers, including 
Table 7 Comparison of the overall success rate between seven traditional models and evolutionary information model on Dataset 1 and Dataset 2, respectively

\begin{tabular}{|c|c|c|}
\hline \multirow[t]{2}{*}{ Method } & Dataset 1 & Dataset 2 \\
\hline & Overall success rate & Overall success rate \\
\hline EDPPSSM+RF & $86.82 \%$ & $79.83 \%$ \\
\hline KPSSM+RF & $92.34 \%$ & $87.99 \%$ \\
\hline CPSSM+RF & $88.83 \%$ & $83.55 \%$ \\
\hline TriPSSM+RF & $93.21 \%$ & $89.43 \%$ \\
\hline RPSSM+RF & $90.56 \%$ & $84.05 \%$ \\
\hline COPSSM+RF & $90.79 \%$ & $84.21 \%$ \\
\hline PsePSSM+RF & $91.71 \%$ & $86.85 \%$ \\
\hline Our model & $94.02 \%$ & $89.79 \%$ \\
\hline
\end{tabular}

Note: Best performing method in bold.
EDPSSM, KPSSM, CPSSM, TriPSSM, RPSSM, CoPSSM and PsePSSM. They are all powerful features extracted from PSSM. Table 7 gives the overall success rate between seven traditional models and the evolutionary information model on Dataset1 and Dataset 2. From the table, we find the best method for the traditional model is TriPSSM, which utilizes PSSM linear probabilities to compute features. Although TriPSSM can obtain the outstanding grades on membrane protein types prediction, we find that it has 8000-dimensional features; the high dimensionality of features in turn increases the computing complexity. Our model is still effective since it can use GPU acceleration to yield better results in less time.

Table 8 Comparison of the Se, Sp, ACC and MCC between seven traditional models and the evolutionary information model on Dataset 1

\begin{tabular}{|c|c|c|c|c|c|c|c|c|c|}
\hline Membrane protein types & Index & PsePSSM & RPSSM & EDPSSM & KPSSM & CPSSM & CoPSSM & TriPSSM & Our mode \\
\hline & Se & 0.9122 & 0.8761 & 0.8491 & 0.9054 & 0.8739 & 0.8806 & 0.9122 & 0.9144 \\
\hline \multirow[t]{4}{*}{ Type I } & Sp & 0.9776 & 0.9733 & 0.9622 & 0.9859 & 0.9666 & 0.9781 & 0.9884 & 0.9920 \\
\hline & ACC & 0.9709 & 0.9633 & 0.9506 & 0.9776 & 0.9571 & 0.9682 & 0.9806 & 0.9841 \\
\hline & MCC & 0.8505 & 0.8112 & 0.7546 & 0.8800 & 0.7856 & 0.8328 & 0.8953 & 0.9129 \\
\hline & Se & 0.7308 & 0.6410 & 0.5256 & 0.6923 & 0.4231 & 0.6923 & 0.8205 & 0.8462 \\
\hline \multirow[t]{4}{*}{ Type II } & $\mathrm{Sp}$ & 0.9927 & 0.9918 & 0.9960 & 0.9913 & 0.9979 & 0.9922 & 0.9929 & 0.9944 \\
\hline & ACC & 0.9880 & 0.9855 & 0.9875 & 0.9859 & 0.9875 & 0.9868 & 0.9898 & 0.9912 \\
\hline & MCC & 0.6819 & 0.6067 & 0.6035 & 0.6339 & 0.5713 & 0.6489 & 0.7424 & 0.7748 \\
\hline & Se & 0.1667 & 0.0 & 0.0 & 0.1667 & 0.0 & 0.0 & 0.1667 & 0.1667 \\
\hline \multirow[t]{4}{*}{ Type III } & $\mathrm{Sp}$ & 1.0 & 0.9998 & 1.0 & 1.0 & 1.0 & 1.0 & 1.0 & 0.9993 \\
\hline & $\mathrm{ACC}$ & 0.9988 & 0.9984 & 0.9986 & 0.9988 & 0.9986 & 0.9986 & 0.9988 & 0.9984 \\
\hline & MCC & 0.4080 & -0.0006 & 0.0 & 0.4080 & 0.0 & 0.0 & 0.4080 & 0.2032 \\
\hline & $\mathrm{Se}$ & 0.4167 & 0.5000 & 0.4167 & 0.6667 & 0.0833 & 0.3333 & 0.6667 & 0.75 \\
\hline \multirow[t]{4}{*}{ Type IV } & $S p$ & 0.9995 & 0.9993 & 1.0 & 0.9995 & 1.0 & 0.9998 & 0.9995 & 0.9995 \\
\hline & ACC & 0.9979 & 0.9979 & 0.9984 & 0.9986 & 0.9975 & 0.9979 & 0.9986 & 0.9982 \\
\hline & MCC & 0.5446 & 0.5763 & 0.6450 & 0.7296 & 0.2883 & 0.5156 & 0.7296 & 0.7828 \\
\hline & $\mathrm{Se}$ & 0.9275 & 0.9394 & 0.9219 & 0.9495 & 0.9305 & 0.9326 & 0.9553 & 0.9593 \\
\hline \multirow[t]{4}{*}{ Mutipass } & $S p$ & 0.9654 & 0.9438 & 0.8305 & 0.9625 & 0.9026 & 0.9682 & 0.9691 & 0.9785 \\
\hline & ACC & 0.9444 & 0.9405 & 0.8994 & 0.9527 & 0.9236 & 0.9414 & 0.9587 & 0.9640 \\
\hline & MCC & 0.8619 & 0.8493 & 0.7360 & 0.8799 & 0.8042 & 0.8560 & 0.8947 & 0.9083 \\
\hline & $\mathrm{Se}$ & 0.3158 & 0.2368 & 0.2105 & 0.3684 & 0.2105 & 0.3684 & 0.3158 & 0.6053 \\
\hline \multirow[t]{4}{*}{ Lipid-chain-anchored } & $\mathrm{Sp}$ & 0.9986 & 0.9979 & 0.9977 & 0.9984 & 0.9991 & 0.9972 & 0.9970 & 0.9960 \\
\hline & ACC & 0.9926 & 0.9912 & 0.9908 & 0.9928 & 0.9922 & 0.9917 & 0.9910 & 0.9926 \\
\hline & MCC & 0.4557 & 0.3403 & 0.3018 & 0.4924 & 0.3719 & 0.4414 & 0.3850 & 0.5862 \\
\hline & $\mathrm{Se}$ & 0.6522 & 0.5217 & 0.3913 & 0.6957 & 0.3913 & 0.7174 & 0.6957 & 0.8043 \\
\hline \multirow[t]{4}{*}{ GPI-anchored } & $\mathrm{Sp}$ & 0.9963 & 0.9986 & 0.9967 & 0.9986 & 0.9988 & 0.9951 & 0.9984 & 0.9963 \\
\hline & ACC & 0.9926 & 0.9935 & 0.9903 & 0.9954 & 0.9924 & 0.9922 & 0.9952 & 0.9942 \\
\hline & MCC & 0.6484 & 0.6431 & 0.4645 & 0.7631 & 0.5503 & 0.6582 & 0.7531 & 0.7465 \\
\hline & $\mathrm{Se}$ & 0.9077 & 0.8536 & 0.6824 & 0.8784 & 0.8176 & 0.8851 & 0.8964 & 0.9009 \\
\hline \multirow[t]{3}{*}{ Peripheral } & $\mathrm{Sp}$ & 0.9537 & 0.9509 & 0.9481 & 0.9524 & 0.9403 & 0.9452 & 0.9578 & 0.9637 \\
\hline & ACC & 0.9490 & 0.9409 & 0.9208 & 0.9448 & 0.9278 & 0.9391 & 0.9515 & 0.9573 \\
\hline & MCC & 0.7655 & 0.7218 & 0.5959 & 0.7427 & 0.6678 & 0.7260 & 0.7711 & 0.7933 \\
\hline
\end{tabular}

Note: Best performing method in bold. 
Tables 8 and 9 report the results, measured in Se, Sp, $\mathrm{ACC}$ and MCC over all methods on Dataset 1 and Dataset 2. From the tables, we can draw a conclusion that our model is more effective than the traditional model in evolution information extraction. The convolutional layer has a powerful ability to extract features from the evolutionary information, and the CapsNet layer can extract local position information. These abilities explain why our DNN model could achieve better results.

The effective of stacking ensemble method

To investigate on how the fusion model impacts the performance, we report the overall success rate of the validation set and testing set using two single models and

Table 9 Comparison of the Se, Sp, ACC and MCC between seven traditional models and the evolutionary information model on Dataset 2

\begin{tabular}{|c|c|c|c|c|c|c|c|c|c|}
\hline Membrane protein types & Index & PsePSSM & RPSSM & EDPSSM & KPSSM & CPSSM & COPSSM & TPSSM & Our model \\
\hline & $\mathrm{Se}$ & 0.8571 & 0.7633 & 0.7265 & 0.7959 & 0.7633 & 0.7673 & 0.8653 & 0.8082 \\
\hline \multirow[t]{4}{*}{ Type I } & $\mathrm{Sp}$ & 0.9586 & 0.9574 & 0.9512 & 0.9705 & 0.9556 & 0.9550 & 0.9759 & 0.9845 \\
\hline & ACC & 0.9517 & 0.9442 & 0.9359 & 0.9587 & 0.9426 & 0.9423 & 0.9684 & 0.9725 \\
\hline & MCC & 0.6943 & 0.6290 & 0.5821 & 0.7048 & 0.6224 & 0.6229 & 0.7748 & 0.7853 \\
\hline & $\mathrm{Se}$ & 0.4304 & 0.4051 & 0.3038 & 0.4810 & 0.2785 & 0.4304 & 0.4557 & 0.4684 \\
\hline \multirow[t]{4}{*}{ Type ॥ } & $\mathrm{Sp}$ & 0.9887 & 0.9881 & 0.9904 & 0.9889 & 0.9952 & 0.0 .9858 & 0.9906 & 0.9901 \\
\hline & ACC & 0.9764 & 0.9753 & 0.9753 & 0.9778 & 0.9795 & 0.9736 & 0.9789 & 0.9786 \\
\hline & MCC & 0.4327 & 0.4059 & 0.3423 & 0.4759 & 0.3873 & 0.4039 & 0.4769 & 0.4797 \\
\hline & $\mathrm{Se}$ & 0.0 & 0.0 & 0.0 & 0.0 & 0.0 & 0.0 & 0.0 & 0.1111 \\
\hline \multirow[t]{4}{*}{ Type III } & $\mathrm{Sp}$ & 1.0 & 1.0 & 1.0 & 1.0 & 1.0 & 1.0 & 1.0 & 0.9958 \\
\hline & ACC & 0.9975 & 0.9975 & 0.9975 & 0.9975 & 0.9975 & 0.9975 & 0.9975 & 0.9936 \\
\hline & MCC & 0.0 & 0.0 & 0.0 & 0.0 & 0.0 & 0.0 & 0.0 & 0.0803 \\
\hline & $\mathrm{Se}$ & 0.1765 & 0.1176 & 0.0588 & 0.3529 & 0.1176 & 0.2353 & 0.3529 & 0.3529 \\
\hline \multirow[t]{4}{*}{ Type IV } & $\mathrm{Sp}$ & 0.9994 & 0.9997 & 1.0 & 1.0 & 1.0 & 1.0 & 1.0 & 0.9980 \\
\hline & ACC & 0.9956 & 0.9956 & 0.9956 & 0.9969 & 0.9958 & 0.9964 & 0.9969 & 0.9950 \\
\hline & MCC & 0.3238 & 0.2788 & 0.2420 & 0.5932 & 0.3423 & 0.4842 & 0.5932 & 0.4011 \\
\hline & $\mathrm{Se}$ & 0.9044 & 0.8971 & 0.8882 & 0.9350 & 0.9023 & 0.8910 & 0.9435 & 0.9443 \\
\hline \multirow[t]{4}{*}{ Mutipass } & $\mathrm{Sp}$ & 0.9494 & 0.9050 & 0.7655 & 0.9263 & 0.8712 & 0.9334 & 0.9316 & 0.9574 \\
\hline & ACC & 0.9184 & 0.8996 & 0.8499 & 0.9323 & 0.8926 & 0.9043 & 0.9398 & 0.9484 \\
\hline & MCC & 0.8232 & 0.7776 & 0.6517 & 0.8464 & 0.7572 & 0.7930 & 0.8627 & 0.8839 \\
\hline & $\mathrm{Se}$ & 0.1111 & 0.0278 & 0.0833 & 0.0833 & 0.0278 & 0.0833 & 0.0833 & 0.6111 \\
\hline \multirow[t]{4}{*}{ Lipid-chain-anchored } & $\mathrm{Sp}$ & 0.9986 & 0.9997 & 0.9997 & 0.9986 & 0.9994 & 0.9992 & 0.9983 & 0.9927 \\
\hline & ACC & 0.9897 & 0.9900 & 0.9906 & 0.9895 & 0.9897 & 0.9900 & 0.9892 & 0.9889 \\
\hline & MCC & 0.2186 & 0.1161 & 0.2480 & 0.1731 & 0.0939 & 0.2012 & 0.1627 & 0.5238 \\
\hline & $\mathrm{Se}$ & 0.4146 & 0.2927 & 0.2439 & 0.4146 & 0.2927 & 0.3902 & 0.4146 & 0.7073 \\
\hline \multirow[t]{4}{*}{ GPI-anchored } & $\mathrm{Sp}$ & 0.9961 & 0.9986 & 0.9983 & 0.9949 & 0.9992 & 0.9983 & 0.9992 & 0.9966 \\
\hline & ACC & 0.9895 & 0.9906 & 0.9897 & 0.9914 & 0.9911 & 0.9881 & 0.9925 & 0.9933 \\
\hline & MCC & 0.4717 & 0.4508 & 0.3864 & 0.5381 & 0.4807 & 0.4226 & 0.5907 & 0.7039 \\
\hline & $\mathrm{Se}$ & 0.8884 & 0.8183 & 0.6581 & 0.8512 & 0.7883 & 0.8326 & 0.8741 & 0.8627 \\
\hline \multirow[t]{3}{*}{ Peripheral } & $\mathrm{Sp}$ & 0.9253 & 0.9050 & 0.9112 & 0.9312 & 0.9046 & 0.9064 & 0.9377 & 0.9404 \\
\hline & ACC & 0.9181 & 0.8882 & 0.8621 & 0.9156 & 0.8821 & 0.8921 & 0.9254 & 0.9254 \\
\hline & MCC & 0.7616 & 0.6742 & 0.5635 & 0.7460 & 0.6513 & 0.6872 & 0.7752 & 0.7725 \\
\hline
\end{tabular}

Note: Best performing method in bold. 
Table 10 Comparison of the overall success rate between two single information model and fusion model on Dataset 1 and Dataset 2

\begin{tabular}{|c|c|c|c|c|}
\hline \multirow{2}{*}{ Method } & \multicolumn{2}{|l|}{ Dataset 1} & \multicolumn{2}{|l|}{ Dataset 2} \\
\hline & Validation set & Testing set & Validation set & Testing set \\
\hline $\begin{array}{l}\text { Sequence } \\
\text { information } \\
\text { model }\end{array}$ & $85.54 \%$ & $91.58 \%$ & $83.25 \%$ & $89.18 \%$ \\
\hline $\begin{array}{l}\text { Evolutionary } \\
\text { information } \\
\text { model }\end{array}$ & $90.46 \%$ & $94.02 \%$ & $80.98 \%$ & $89.79 \%$ \\
\hline Fusion model & - & $95.68 \%$ & - & $92.98 \%$ \\
\hline
\end{tabular}

Note: Best performing method in bold.

the fusion model (Table 10). From the table, we find that the fusion model achieves better results both on datasets, demonstrating its superiority in prediction.

Figures 7 and 8 give the comparison results, measured in Se, Sp, ACC and MCC over three models on Dataset 1 and Dataset 2,respectively. We find that the fusion model achieves the best results in most cases. However, the performance of small-scale forecasts has not yet met our expectations. Other effective strategies for solving imbalance problems may contribute to improving our method.

Furthermore, the prediction of membrane protein types requires not only high classification accuracy but also certain operational efficiency. Table 11 lists the running time for different models when predicting 100 membrane protein samples. We find that the process of fusion takes approximately $1.93 \mathrm{e}-10 \mathrm{~s}$, and the running time of the fusion model is almost the sum of two single models. Though the time complexity of the fusion model is higher, it is also within the tolerance range.

\section{Comparison with the existing methods}

To demonstrate the performance of our DNN model in practical use, we compared our DNN model with eight state-of-the-art machine learning methods in the prediction of membrane protein types. These models all proceed through complex feature engineering, including feature extraction, dimension reduction and so on. Table 12 represents the overall success rate between our DNN model with eight models on Dataset 1 and Dataset 2. Taking the accuracy of prediction and the degree of domain experts dependence into account, we find the DNN model not only achieves the best performance both on Dataset 1 and Dataset 2, but also removes the burden of feature engineering, which illustrates the effectiveness of our model.

\section{Conclusion}

In this paper, we propose two DNN models to process the sequence information and evolutionary information separately. Among these models, 1D convolutional layers and Bi-LSTM layers are used for the sequence information model, while the evolutionary information model

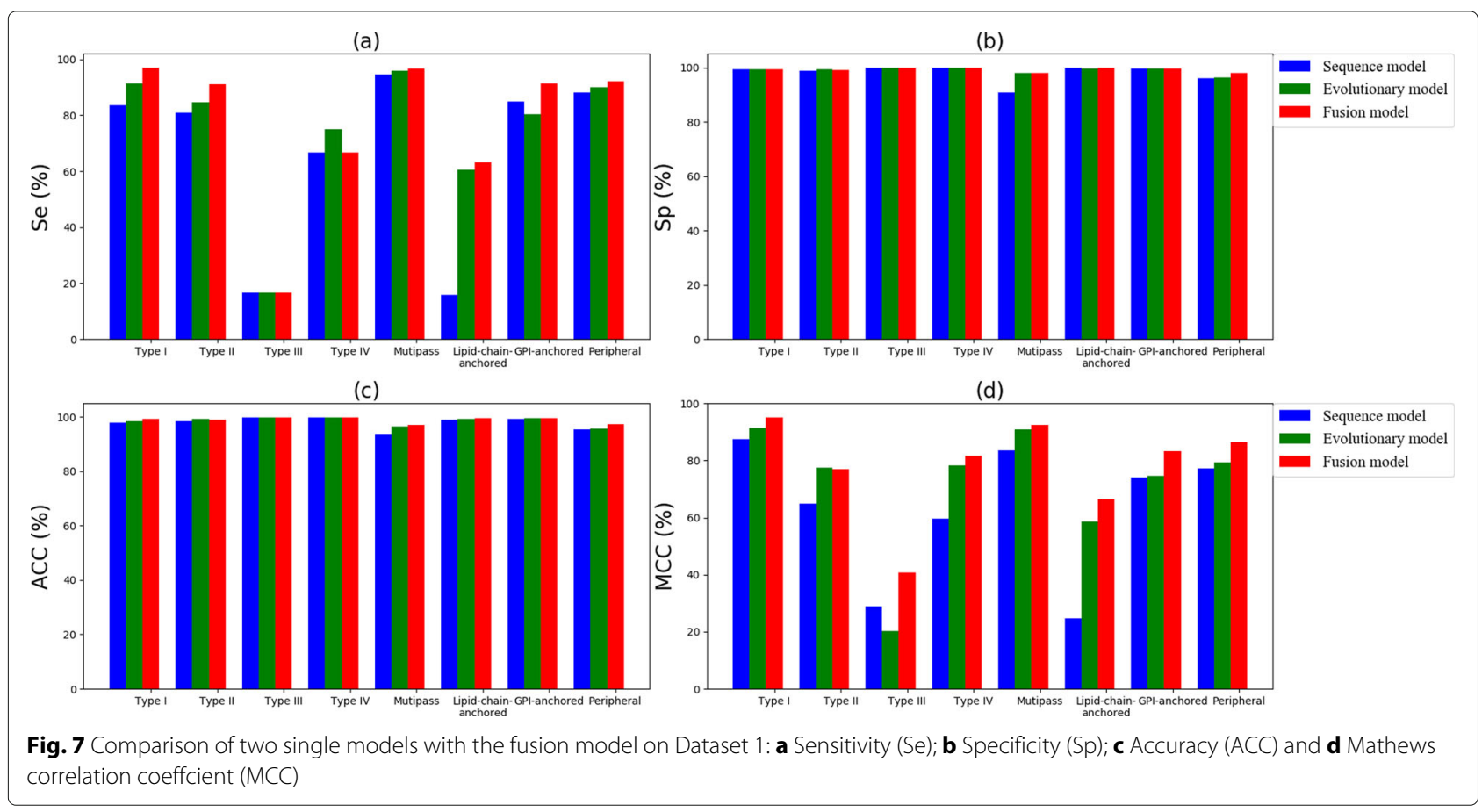




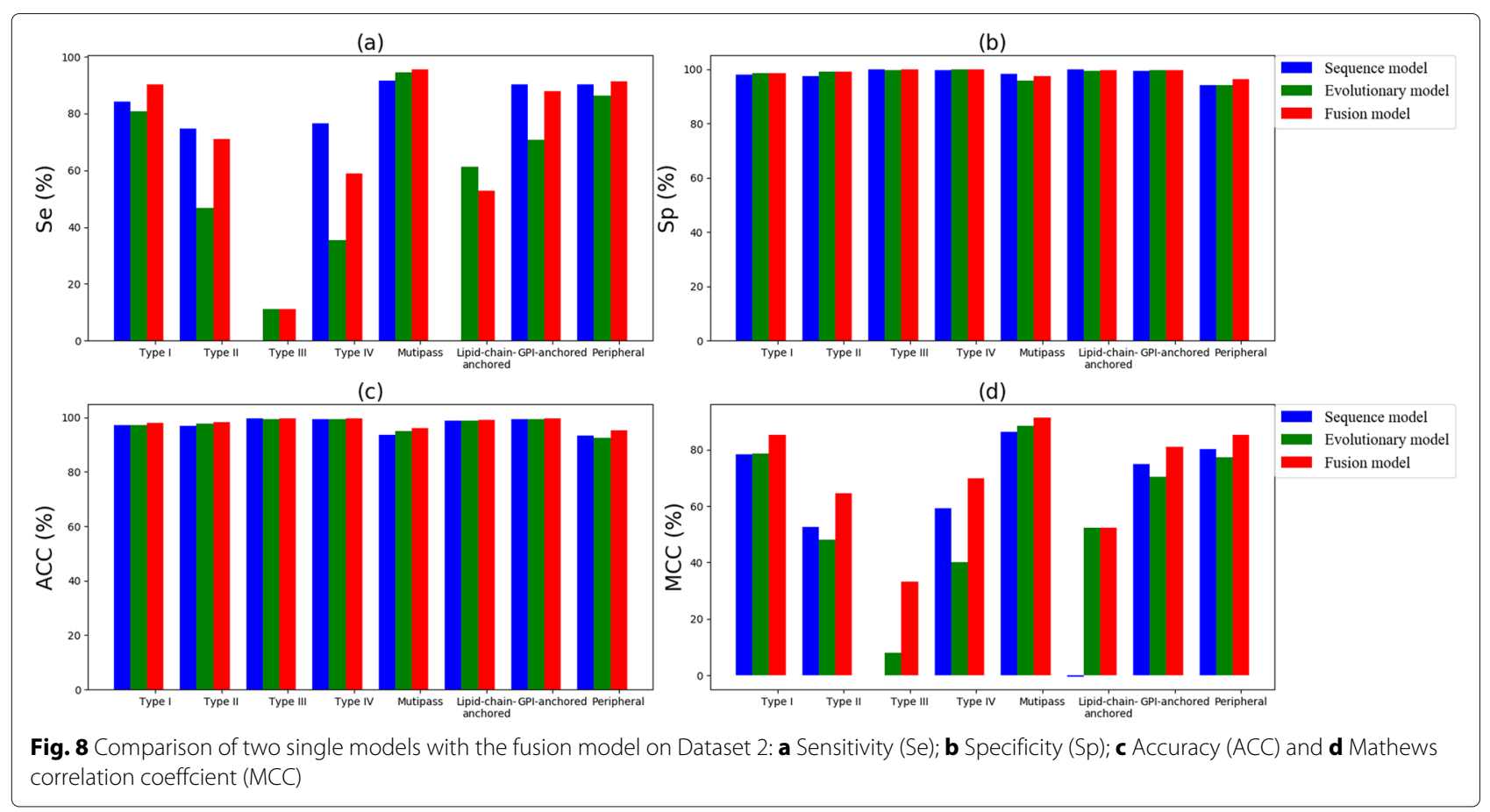

uses 2D convolutional layers and CapsNet layers. In the comparative experiments, we compare these models with the traditional model. Numerous experimental results demonstrate that our two proposed DNN models not only obtain more competitive results in membrane protein type prediction but also can remove the burden of feature engineering and the reliance on domain experts. In addition, to obtain a better prediction performance, the stacking ensemble method is used to fuse the above two models.

Furthermore, we provide a new vector representation method based on autoencoder and the physicochemical properties of amino acids to improve the performance of the sequence information model. Compared with the one-hot encoding, the new vector representation method not only represents the relationship between amino acids well but also can effectively improve the prediction performance.

Finally, our method can be applied not only to membrane protein type prediction but also to other fields

Table 11 Comparison of the running time for different models when predicting 100 membrane protein samples

\begin{tabular}{ll}
\hline Model & Running time (s) \\
\hline Sequence information model & 0.97 \\
Evolutionary information model & 0.50 \\
Fusion model & 1.47
\end{tabular}

of bioinformatics [51-63]. However, there is still room for further investigation. For example, the problem of an imbalanced dataset had a negative effect on the accuracy of small-sized types. Some strategies for processing with the imbalanced dataset may improve the performance of our DNN model.

Table 12 Comparison of the overall success rate with eight state-of-the-art methods

\begin{tabular}{lll}
\hline \multirow{2}{*}{ Method } & \multicolumn{2}{l}{ Overall success rate } \\
\cline { 2 - 3 } & Dataset 1 & Dataset 2 \\
\hline AAC based on Covariance [9] & $37.2 \%$ & - \\
$\begin{array}{l}\text { PsePSSM based on ensemble } \\
\text { method [9] }\end{array}$ & $91.6 \%$ & $78.3 \%$ \\
$\begin{array}{l}\text { Physicochemical properties } \\
\text { based on ensemble method }\end{array}$ & $91.0 \%$ & - \\
$\begin{array}{l}\text { [13] } \\
\begin{array}{l}\text { Fusion representation based } \\
\text { on SVM [16] }\end{array}\end{array}$ \\
$\begin{array}{l}\text { PsePSSM based on LLDA and } \\
\text { ensemble method [22] }\end{array}$ & & \\
$\begin{array}{l}\text { PsePSSM and DC based on GPP } \\
\text { and KNN [20] }\end{array}$ & $92.6 \%$ & - \\
$\begin{array}{l}\text { PsePSSM based on PCA and } \\
\text { KNN [22] }\end{array}$ & $98.7 \%$ & - \\
$\begin{array}{l}\text { Previous work [19] } \\
\text { This paper }\end{array}$ & $90.2 \%$ & - \\
\hline
\end{tabular}

Note: Best performing method in bold. 


\section{Abbreviations}

ACC: accuracy; AD: autocorrelation descriptor; Bi-LSTM: bidirectional long short term memory; CBOW: Continuous bag of words; CoPSSM: correlation position-specific scoring matrix; DipC: dipeptide composition; DNN: deep neural network; EDPSSM: evolutionary difference position-specific score matrix; FN: false negative; FP: false positive; GD: global descriptor; HHT: Hilbert-Huang transform; KNN: k-nearest neighbor; KPSSM: k-separated-bigrams position-specific score matrix; LAAC: Local amino acid composition; LDC: local dipeptide composition; LZC: Lempel-Ziv complexity; MCC: Matthews correlation coefficient; MLR: multiple logistic regression. NBM: naive bayesian model; NLP: natural language processing; OSR: overall success rate; PNN: probabilistic neural network; PseAAC: pseudo-amino acid composition; PsePSSM: pseudo position-specific score matrix; PSSM: position-specific scoring matrix; RF: random forests; RPSSM: reduced position-specific score matrix; SD: sequence-order descriptor; Se: sensitivity; Sp: specificity; SVM: support vector machines; TipC: tripeptide composition; TN: true negative; TP: true positive; TriPSSM: tri-gram position-specific score matrix

\section{Acknowledgement}

The authors would like to thank the reviewers and editors for their patient guidance and valuable suggestions.

\section{About this supplement}

This article has been published as part of BMC Bioinformatics Volume 20 Supplement 25, 2019: Proceedings of the 2018 International Conference on Intelligent Computing (ICIC 2018) and Intelligent Computing and Biomedical Informatics (ICBI) 2018 conference: bioinformatics. The full contents of the supplement are available online at https://bmcbioinformatics.biomedcentral. com/articles/supplements/volume-20-supplement-25.

\section{Authors' contributions}

LG and SW conceived the algorithm, prepared the datasets, carried out experiments. LG wrote the manuscript. SW, ML, ZC provided some suggestions for the manuscript writing and revised it critically for important intellectual content. All authors read and approved the final manuscript.

\section{Funding}

This research is supported by grants from National Natural Science Foundation of China (11661081), Natural Science Foundation of Yunnan Province (2017FA032) and Training Plan for Young and Middle-aged Academic Leaders of Yunnan Province (2018HB031).

\section{Availability of data and materials}

The related source codes and datasets are available at https:/github.com/ DragonKnightss/MembraneProteinTypePrediction.

\section{Ethics approval and consent to participate}

Not applicable.

\section{Consent for publication}

Not applicable.

\section{Competing interests}

The authors declare that they have no competing interests.

\section{Author details}

${ }^{1}$ Department of Computer Science and Engineering, School of Information Science and Engineering, Yunnan University, Kunming 650504, People's Republic of China. ${ }^{2}$ School of Public Health (Shenzhen), Sun Yat-sen University, Guangzhou 510006, People's Republic of China.

\section{Published: 24 December 2019}

\section{References}

1. Deng SP, Huang DS. SFAPS: An R package for structure/function analysis of protein sequences based on informational spectrum method. Methods. 2014;69(3):207-12.

2. Huang DS, Yu HJ. Normalized Feature Vectors: A Novel Alignment-Free Sequence Comparison Method Based on the Numbers of Adjacent Amino Acids. IEEE/ACM Trans Comput Biol Bioinforma. 2013;10(2):457-67.
3. Xia JF, Zhao XM, Song J, et al. APIS: accurate prediction of hot spots in protein interfaces by combining protrusion index with solvent accessibility. BMC Bioinformatics. 2010;11(1):174-0.

4. Wang B, Chen P, Huang DS, et al. Predicting protein interaction sites from residue spatial sequence profile and evolution rate. Febs Lett. 2006;580(2):0-384.

5. Zhao XM, Cheung YM, Huang DS. A novel approach to extracting features from motif content and protein composition for protein sequence classification. Neural Netw. 2005;18(8):1019-28.

6. Almén MS, Nordström KJV, Fredriksson R, et al. Mapping the human membrane proteome: a majority of the human membrane proteins can be classified according to function and evolutionary origin. BMC Biol. 2009;7(1):50.

7. Krogh A, Larsson B, Heijne GV, et al. Predicting transmembrane protein topology with a hidden markov model: application to complete genomes. J Mol Biol. 2001;305(3):0-580.

8. Overington JP, Al-Lazikani B, Hopkins AL. How many drug targets are there? Nat Rev Drug Discov. 2006;5(12):993-6.

9. Chou KC, Shen HB. MemType-2L: A Web server for predicting membrane proteins and their types by incorporating evolution information through Pse-PSSM. Biochem Biophys Res Commun. 2007;360(2):0-345.

10. Wang $L$, Yuan $Z$, Chen $X$, et al. The prediction of membrane protein types with NPE. IEICE Electron Express. 2010;7(6):397-402.

11. Ali F, Hayat M. Classification of membrane protein types using Voting Feature Interval in combination with Chou's Pseudo Amino Acid Composition. J Theor Biol. 2015;384:78-83.

12. Chen YK, Li KB. Predicting membrane protein types by incorporating protein topology, domains, signal peptides, and physicochemical properties into the general form of Chou's pseudo amino acid composition. J Theor Biol. 2013;318:1-12.

13. Nanni L, Lumini A. An ensemble of support vector machines for predicting the membrane protein type directly from the amino acid sequence. Amino Acids. 2008;35(3):573-80.

14. Wan S, Mak MW, Kung SY. Mem-ADSVM: A two-layer multi-label predictor for identifying multi-functional types of membrane proteins. J Theor Biol. 2016;398(7):32-42.

15. Hayat M, Khan A. Mem-PHybrid: Hybrid features-based prediction system for classifying membrane protein types. Anal Biochem. 2012;424(1):35-44.

16. Hayat $M$, Khan A. Predicting membrane protein types by fusing composite protein sequence features into pseudo amino acid composition. J Theor Biol. 2011;271(1):10-7.

17. Chou KC. Prediction of protein cellular attributes using pseudo-amino acid composition. Proteins-Struct Funct Bioinforma. 2010;43(3):246-55.

18. Han GS, Yu ZG, Anh V. A two-stage SVM method to predict membrane protein types by incorporating amino acid classifications and physicochemical properties into a general form of Chou's PseAAC. J Theor Biol. 2014;344:31-9.

19. Guo L, Wang S. Prediction for Membrane Protein Types Based on Effective Fusion Representation and MIC-GA Feature Selection. IEEE Access. 2018;6:75669-81.

20. Wang T, Xia T, Hu XM. Geometry preserving projections algorithm for predicting membrane protein types. J Theor Biol. 2010;262(2):208-13.

21. Anishetty $S$, Pennathur $G$, Anishetty R. Tripeptide analysis of protein structures. BMC Struct Biol. 2002;2(1):9.

22. Wang T, Yang J, Shen HB, et al. Predicting membrane protein types by the LLDA algorithm. Protein Pept Lett. 2008;15(9):915-21.

23. Ding S, Li Y, Shi Z, et al. A protein structural classes prediction method based on predicted secondary structure and PSI-BLAST profile. Biochimie. 2014;97(2):60-5.

24. Zhang L, Zhao X, Kong L. Predict protein structural class for low-similarity sequences by evolutionary difference information into the general form of Chou's pseudo amino acid composition. J Theor Biol. 2014;355:105-10.

25. Sharma A, Lyons J, Dehzangi A, et al. A feature extraction technique using bi-gram probabilities of position specific scoring matrix for protein fold recognition. J Theor Biol. 2013;320:41-6.

26. Harsh S, Gaurav R, et al. Protein Fold Recognition Using Genetic Algorithm Optimized Voting Scheme and Profile Bigram. J Softw. 2016;1 (8):756-67.

27. Wang $S$, Yue Y. Protein subnuclear localization based on a new effective representation and intelligent kernel linear discriminant analysis by dichotomous greedy genetic algorithm. Plos ONE. 2018;13(4):e0195636. 
28. Patel S, Tripathi R, Kumari V, et al. Deeplnteract: Deep Neural Network based Protein-Protein Interaction prediction tool. Current Bioinformatics. 2017;12(6):. https://doi.org/10.2174/1574893611666160815150746.

29. Veltri D, Kamath U, Shehu A. Deep Learning Improves Antimicrobial Peptide Recognition. Bioinformatics. 2018;34(16):2740-7.

30. Sun S, Wu Q, Peng Z, et al. Enhanced prediction of RNA solvent accessibility with long short-term memory neural networks and improved sequence profiles. Bioinformatics. 2019;35(10):1686-91.

31. Zhang Z, Zhao Y, et al. Deep learning in omics: a survey and guideline. Brief Funct Genom. 2019;8(1):41-57.

32. Guohui C, Hanhui M, Jifang Y, et al. DeepCRISPR: optimized CRISPR guide RNA design by deep learning. Genome Biol. 2018;19(1):88.

33. Wei L, Ding Y, Su R, et al. Prediction of human protein subcellular localization using deep learning. J Parallel Distrib Comput. 2017;117:212-7.

34. Derevyanko G, Grudinin S, et al. Deep convolutional networks for quality assessment of protein folds. Bioinformatics. 2018;34(23):4046-53.

35. Luo F, Wang M, et al. DeepPhos: prediction of protein phosphorylation sites with deep learning. Bioinformatics. 2019:35(16):2766-73.

36. Nie L, Deng L, Fan C, et al. Prediction of Protein S-Sulfenylation Sites Using a Deep Belief Network. Curr Bioinforma. 2018;13(5):461-7.

37. Shen Z, Bao W, et al. Recurrent neural network for predicting transcription factor binding sites. Sci Rep. 2018;8:15270.

38. Lecun Y, Bengio Y, Hinton G. Deep learning. Nature. 2015;521(7553):436.

39. Graves A, Schmidhuber J. Framewise phoneme classification with bidirectional LSTM and other neural network architectures. Neural Netw. 2005;18(5):602-10.

40. Sabour S, Frosst N, Hinton GE. Dynamic routing between capsules. Advances in Neural Information Processing Systems. 2017;3859-69.

41. Wan S, Mak MW, Kung SY. Benchmark data for identifying multi-functional types of membrane proteins. Data Brief. 2016;8(C):105-7.

42. Jones DT. Protein secondary structure prediction based on position-specific scoring matrices. J Mol Biol. 1999;292(2):0-202.

43. Bin $L$, Kai $L$, De-Shuang $H$, et al. iEnhancer-EL: Identifying enhancers and their strength with ensemble learning approach. Bioinformatics. 2018;34(22):3835-42.

44. Wang B, San Wong H, Huang DS. Inferring Protein-Protein Interacting Sites Using Residue Conservation and Evolutionary Information. Protein Pept Lett. 2006;13(10):999-1005.

45. Xia J, Zhao X, et al. Predicting protein-protein interactions from protein sequences using meta predictor. Amino Acids. 2010;39(5):1595-9.

46. Ting KM, Witten IH. Issues in stacked generalization. J Artif Intell Res. 2011;10(1):271-89.

47. Powers DMW. Evaluation: from precision, recall and f-factor to roc, informedness, markedness \& correlation. J Mach Learn Technol. 2011;2(1) 37-63.

48. Mikolov T, Chen K, Corrado G, Dean J. Efficient estimation of word representations in vector space. 2013. arXiv:1301.3781.

49. Kawashima S, Ogata H, Kanehisa M. AAindex: Amino Acid Index Database. Nucleic Acids Res. 1999;27(1):368-9.

50. Sankari ES, Manimegalai D. Predicting membrane protein types using various decision tree classifiers based on various modes of general PseAAC for imbalanced datasets. J Theor Biol. 2017;435:208-17.

51. Zhu L, Deng SP, You ZH, et al. Identifying Spurious Interactions in the Protein-Protein Interaction Networks Using Local Similarity Preserving Embedding. IEEE/ACM Trans Comput Biol Bioinforma. 2017;14(2):345-52.

52. Zou Q, Xing PW, Wei LY, et al. Gene2vec: gene subsequence embedding for prediction of mammalian N-6-methyladenosine sites from mRNA. RNA. 2019;25(2):205-18.

53. Bin L, Fan W, De-Shuang $H$, et al. iRO-3wPseKNC: identify DNA replication origins by three-window-based PseKNC. Bioinformatics. 2018;34(18): 3086-93.

54. Liu B, Yang F, Huang DS, et al. iPromoter-2L: a two-layer predictor for identifying promoters and their types by multi-window-based PseKNC. Bioinformatics. 2018;4(1):33-40.

55. Guo WL, Huang DS. An efficient method to transcription factor binding sites imputation via simultaneous completion of multiple matrices with positional consistency. Mol BioSyst. 2017;13(9):1827-37.

56. You ZH, Yin Z, Han K, et al. A semi-supervised learning approach to predict synthetic genetic interactions by combining functional and topological properties of functional gene network. BMC Bioinformatics. 2010;11(1):343-0.
57. Chen P, Han K, Li X, et al. Predicting Key Long-Range Interaction Sites by B-Factors. Protein Pept Lett. 2008;15(5):478-83.

58. Huang DS, Zheng $\mathrm{CH}$. Independent component analysis-based penalized discriminant method for tumor classification using gene expression data. Bioinformatics. 2006;22(15):1855-62.

59. Huang DS, Zhang L, Han K, et al. Prediction of Protein-Protein Interactions Based on Protein-Protein Correlation Using Least Squares Regression. Curr Protein Pept Sci. 2014;15(6):553-60.

60. You ZH, Lei YK, Gui J, et al. Using manifold embedding for assessing and predicting protein interactions from high-throughput experimental data. Bioinformatics. 2010;26(21):2744-51.

61. Shi MG, Xia JF, Li XL, et al. Predicting protein-protein interactions from sequence using correlation coefficient and high-quality interaction dataset. Amino Acids. 2010;38(3):891-9.

62. Xia JF, Han K, Huang DS. Sequence-Based Prediction of Protein-Protein Interactions by Means of Rotation Forest and Autocorrelation Descriptor. 17. 2010;1:137-45.

63. Cui G, Chen Y, Huang DS, et al. An Algorithm for Finding Functional Modules and Protein Complexes in Protein-Protein Interaction Networks. Journal of Biomedicine \& Biotechnology. 2014;2008(1110-7243):860270.

\section{Publisher's Note}

Springer Nature remains neutral with regard to jurisdictional claims in published maps and institutional affiliations.

Ready to submit your research? Choose BMC and benefit from:

- fast, convenient online submission

- thorough peer review by experienced researchers in your field

- rapid publication on acceptance

- support for research data, including large and complex data types

- gold Open Access which fosters wider collaboration and increased citations

- maximum visibility for your research: over $100 \mathrm{M}$ website views per year

At BMC, research is always in progress.

Learn more biomedcentral.com/submissions 